\title{
Der Botanische Garten von Orotava auf Teneriffa
}

\author{
Veit Martin Dörken \& Hilke Steinecke
}

\begin{abstract}
The botanical garden in Orotava in Tenerife (Botánico) was designed as an acclimatization garden in the $18^{\text {th }}$ century. Plants from tropical areas were cultivated there in an attempt for adapting them to the specific climatic conditions prevailing in Spain. Later, some of these were planted at the Spanish court in Madrid. Accordingly, the garden still contains many old specimens, including a gigantic fig tree (Ficus macrophylla ssp. columnaris) with profusive aerial roots. The history of the garden and its unique collection are outlined here.
\end{abstract}

\section{Zusammenfassung}

Der Botanische Garten in Orotava auf Teneriffa (Botánico) wurde im 18. Jahrhundert als Akklimatisierungsgarten angelegt. Pflanzen aus tropischen Gebieten sollten an das Klima in Spanien gewöhnt werden und einige von ihnen später am spanischen Hof in Madrid ausgepflanzt werden. Dementsprechend enthält der Garten viele alte Gehölze, darunter einen riesigen Feigenbaum (Ficus macrophylla ssp. columnaris) mit vielen Luftwurzeln. Die Geschichte des Gartens und sein Pflanzenbestand werden vorgestellt.

\section{Einleitung}

Offiziell heißt der Garten Jardín de aclimatación de la Orotava oder auch Jardin Botánico de la Orotava (kurz Botánico). Er befindet sich in Puerto de la Cruz, dem alten Hafen von La Orotava auf Teneriffa. Dieser historische Botanische Garten beeindruckt durch seine Vielzahl exotischer Gewächse, seine zahlreichen großen Palmen und durch seinen alten Baumbestand. Eines der beliebtesten Fotomotive ist sicherlich die riesige Würgefeige (Ficus macrophylla ssp. columnaris) mit ihren ausladenden Luftwurzeln. Der Garten ist deshalb auch eine gut frequentierte Touristenattraktion.

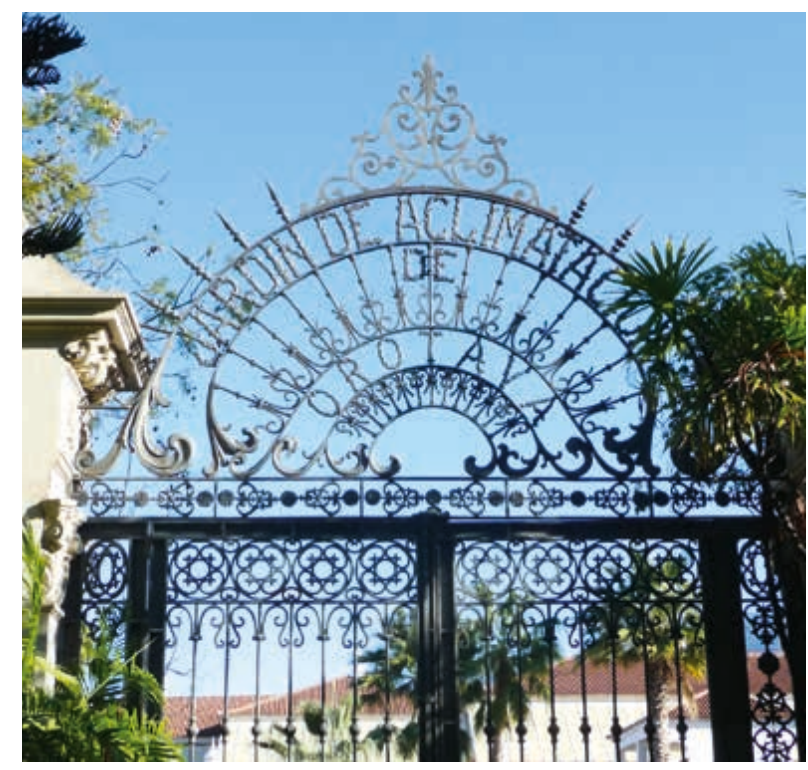

Abb. 1: Eingangstor zum Botanischen Garten.
Jährlich kommen etwa 400000 Besucher in den rund 6 Hektar großen Garten.

\section{Anfänge des Gartens}

Im 18. Jahrhundert begeisterten sich Regenten und Adelige zunehmend für die exotische Pflan-

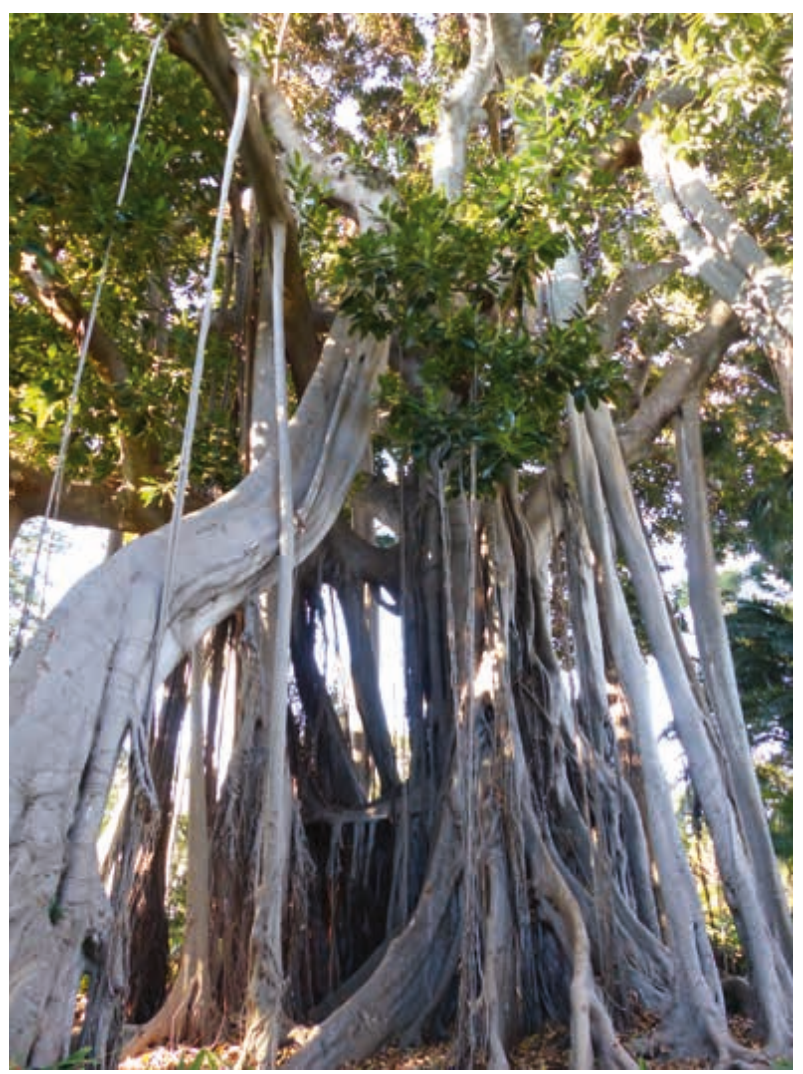

Abb. 2: Die alte Würgefeige (Ficus macrophylla ssp. columnaris). 
zenwelt. Es kam der Wunsch auf, botanische Kostbarkeiten und seltene Exoten auch im eigenen Land in öffentlichen Parkanlagen und in privaten Gärten zu zeigen. Pflanzenjäger wurden in die Welt geschickt, um neue, exotische Pflanzen zu sammeln. Die Seefahrernation Spanien hatte viele Gelegenheiten, aus ihren Kolonien in Übersee Pflanzen mitzubringen.

König Karl (CARLOS) III. beauftragte im Jahr 1788 die Anlage eines Akklimatisierungsgartens. Die Pflanzen sollten hier allmählich an das Klima in Spanien gewöhnt werden, um dann später auf das spanische Festland in den Königlichen Gärten von Madrid gepflanzt zu werden. Der Botánico ist nach dem Botanischen Garten Madrid der zweitälteste Botanische Garten Spaniens. Der Standort für den Botánico in Orotava wurde vor allem aus klimatischen Gründen ausgewählt, da hier aufgrund der Meeresnähe ein ausgeglichenes, mildes Klima vorherrscht, mit dem viele tropische Pflanzen gut zurecht kommen. Alonso de Nava y Grimón, der den Garten anlegte, wurde auch erster Gartenleiter.

König KarL III. konnte die Fertigstellung des Gartens nicht mehr miterleben, aber auch sein Nachfolger KarL IV. begeisterte sich für die Idee des Gartens. Anfang der 1790er-Jahre erfolgten die ersten Bepflanzungen. Der Garten war streng geometrisch geplant. Die Pflanzen wurden nach dem damals gerade erst neu erschienenen System von Linné nach wissenschaftlichen Aspekten angeordnet, weniger nach gestalterischen Gesichtspunkten oder Standortbedürfnissen der Pflanzen. Der Garten war und ist daher immer noch Anziehungspunkt für viele Wissenschaftler. Tätig war hier z. B. auch der französische Naturforscher $\mathrm{S}_{\mathrm{A}-}$ Bin Berthelot (1794-1880), der sich um die Naturgeschichte der Kanaren verdient gemacht hat. Nach ihm benannt ist auch der Kanarische Hornkleee (Lotus berthelothii, Fabaceae), ein vom Aussterben bedrohter Endemit Teneriffas, der sich aber in der Gartenkultur als Zierpflanze gut etabliert hat.

Alexander von Humboldt besuchte im Juni 1799 auf einem dreitägigen Zwischenhalt auf

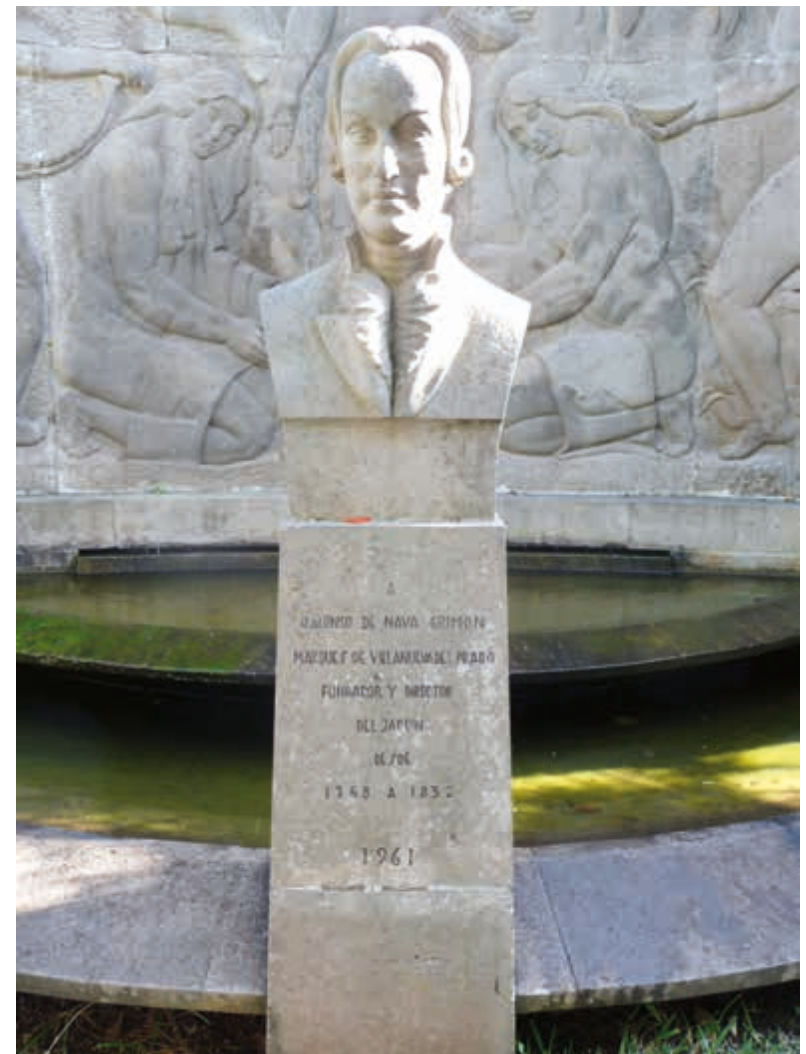

Abb. 3: Büste von Alonso de Nava y Grimón, der den Garten ursprünglich anlegte.

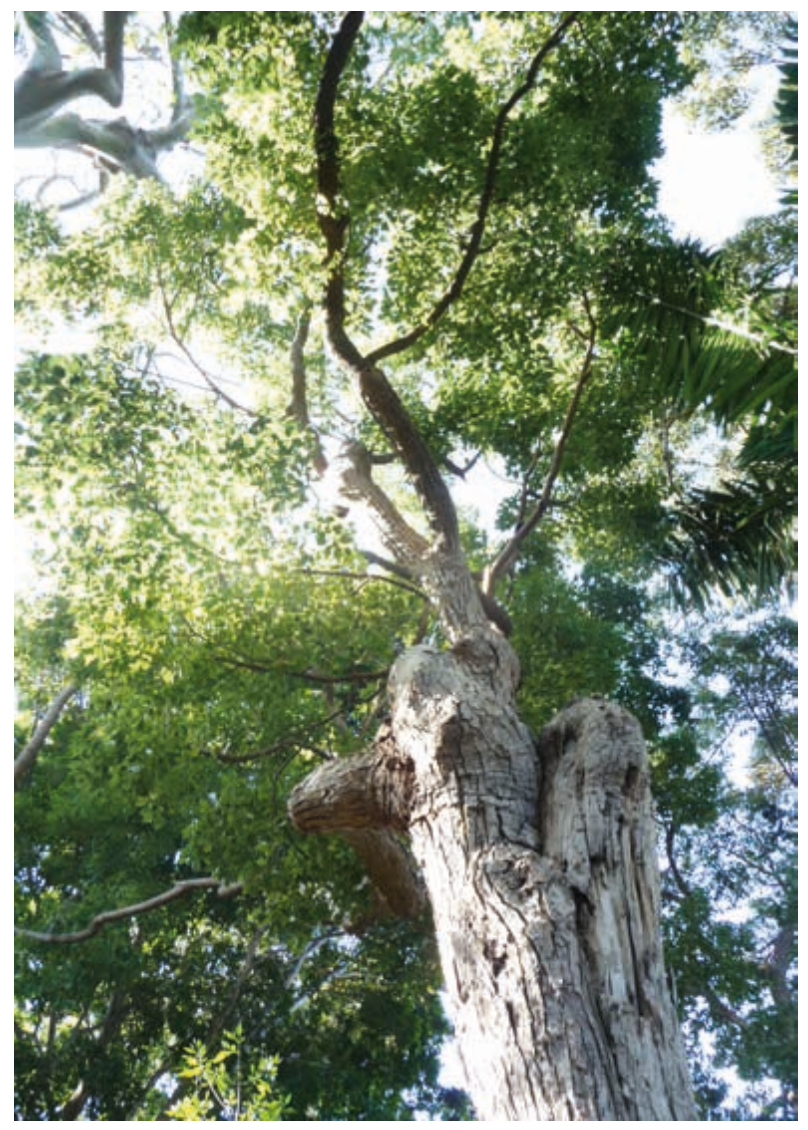

Abb. 4: Im Garten gibt es noch einige alte Gehölze wie auch diesen Kampferbaum (Cinnamomum camphora). 


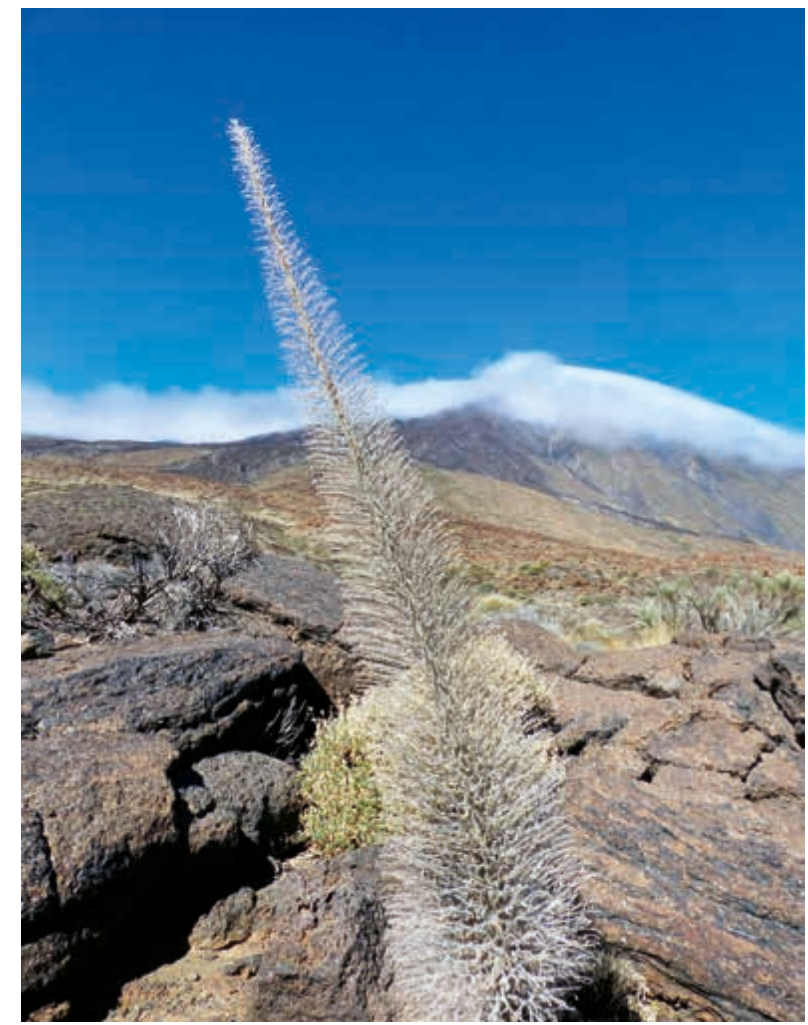

Abb. 5: Alter Fruchtstand des in den Cañadas heimischen Natternkopfes Echium wildpretii, der seinen Namen zu Ehren von Hermann WiLdPret erhalten hat. dem Weg nach Südamerika die Insel Teneriffa, um den Teide zu besteigen. Dabei hatte er auch die Möglichkeit, den Garten zu besuchen und ihn in seiner ersten Glanzzeit kennenzulernen, bevor er nach dem Tod von Alonso de Nava y Grimón 1832 zunehmend verwahrloste. Humboldt schreibt in seinem Reisebericht (Gebauer 2009): „Die Anlage eines botanischen Gartens auf Teneriffa ist ein sehr glücklicher Gedanke, da derselbe sowohl für die wissenschaftliche Botanik als für die Einführung nützlicher Gewächse in Europa sehr förderlich werden kann. Die erste Idee eines solchen verdankt man dem Marquis von Nava, einem Mann, der Poivre an die Seite gestellt zu werden verdient und im Triebe, das Gute zu fördern, von seinem Vermögen den edelsten Gebrauch gemacht hat. Mit ungeheuren Kosten ließ er den Hügel von Durasno, der amphitheathralisch aufsteigt, abheben, und im Jahr 1795 machte man mit Anpflanzungen den Anfang ...

In Durasno wachsen Proteen, der Gojavebaum, der Jambusenbaum, die Chirimoya aus Peru, Mi-

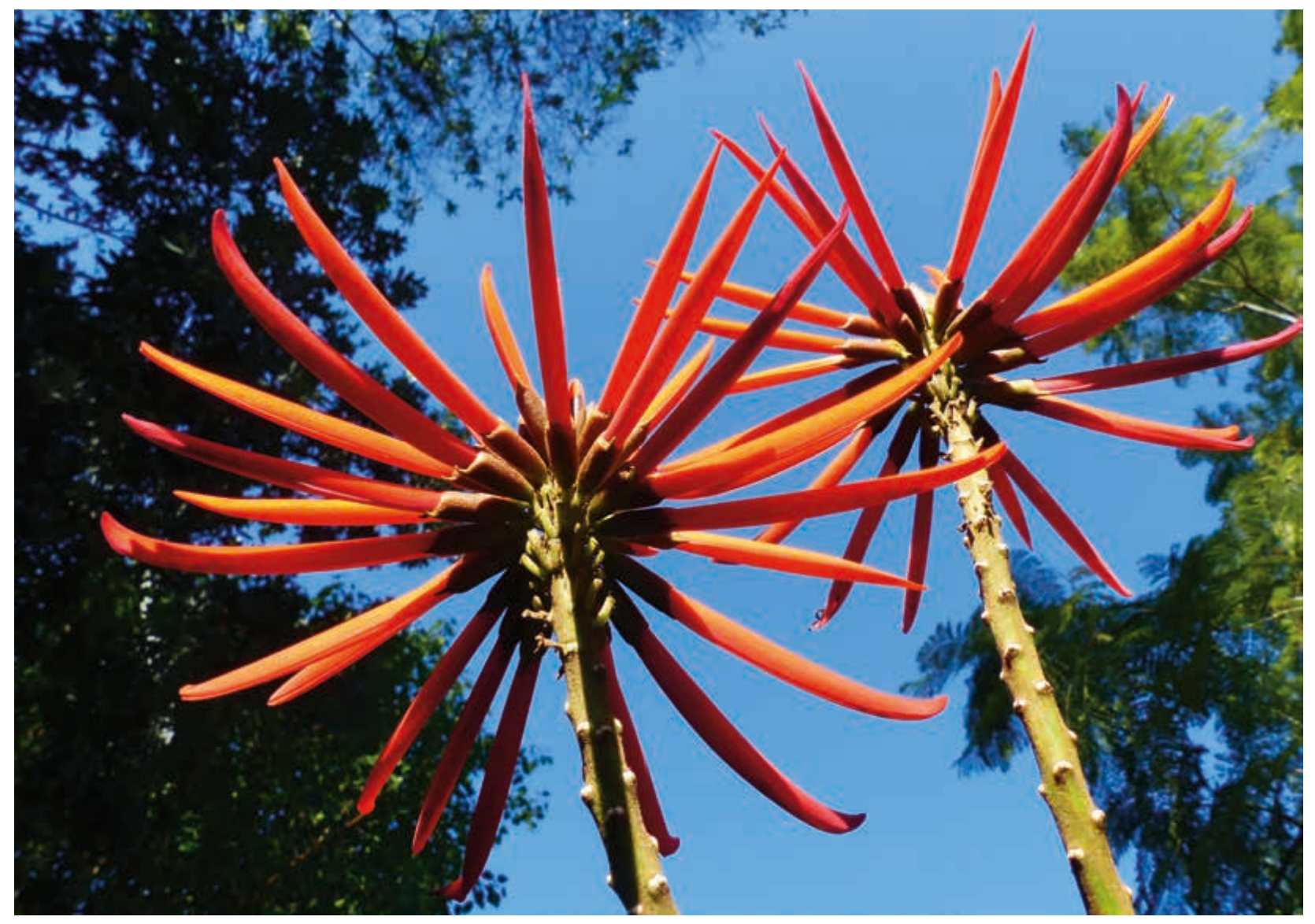

Abb. 6: Der Korallenstrauch (Erythrina speciosa) blüht im Winter, wenn viele andere Arten noch eine Ruhepause machen. 
mosen und Heliconien im Freien. Wir pflückten reife Samen von mehreren schönen Glycinearten aus Neuholland, welche der Gouverneur von $\mathrm{Cu}-$ mana, EMPARAN, mit Erfolg angepflanzt hat und die seitdem auf den südamerikanischen Küsten wild geworden sind.“

\section{Hermann Wildpret}

Die bekannteste Pflanze Teneriffas ist sicherlich der Rote- bzw. Teide-Natternkopf aus der Familie der Raublattgewächse, der in den Cañadas am Teide sowie auf La Palma heimisch ist. Im Mai entwickelt das zweijährige Raublattgewächs seinen roten kerzenförmigen Blütenstand mit Zehntausenden roter Blüten. Im Winter sind in der braunen Lavalandschaft die hellen alten Fruchtstände sehr markant, die wie überdimensionierte Fischgerippe wirken. Diese Nationalblume Teneriffas heißt wissenschaftlich Echium wildpretii, eingedeutscht auch Wildprets Natternkopf. Die Bezeichnung ehrt den Schweizer Gärtner Hermann WildPRET, dessen Leben eng mit dem Botánico verbunden ist. Denn er arbeitete dort von 1860-1893 als
Obergärtner und verhalf durch sein Wirken dem Garten zu einer neuen Blütezeit. Schon wenige Jahre nach seinem Amtsantritt hatte er den Pflanzenbestand um das Zehnfache auf rund $2500 \mathrm{Ar}-$ ten erhöht. WiLDPRET beschäftigte sich auch mit der endemischen Flora Teneriffas und stand im regen Austausch mit anderen Gärten in Europa. Dadurch wurde der Botánico in der 2. Hälfte des 19. Jahrhunderts weltberühmt. Einige der Gehölze, die schon in damaligen Reisebeschreibungen häufig erwähnt wurden, haben bis heute überlebt wie die bereits erwähnte Würgefeige, ein Leberwurstbaum (Kigelia africana), Araukarien und Kanarische Kiefern (Pinus canariensis). Die berühmte Zweihäusige Kermesbeere (Phytolacca dioica) mit ihrem dicken Fuß, die noch bis vor wenigen Jahren ein beliebtes Motiv für Gruppenfotos war, ist leider mittlerweile in sich zusammengebrochen und entfernt.

Wildprets Liebe zur Botanik hat sich übrigens auch in nachfolgenden Generationen erhalten. Hermann Wildprets Enkel Wolfredo Wildpret de la Torre (geb. 1933 in Santa

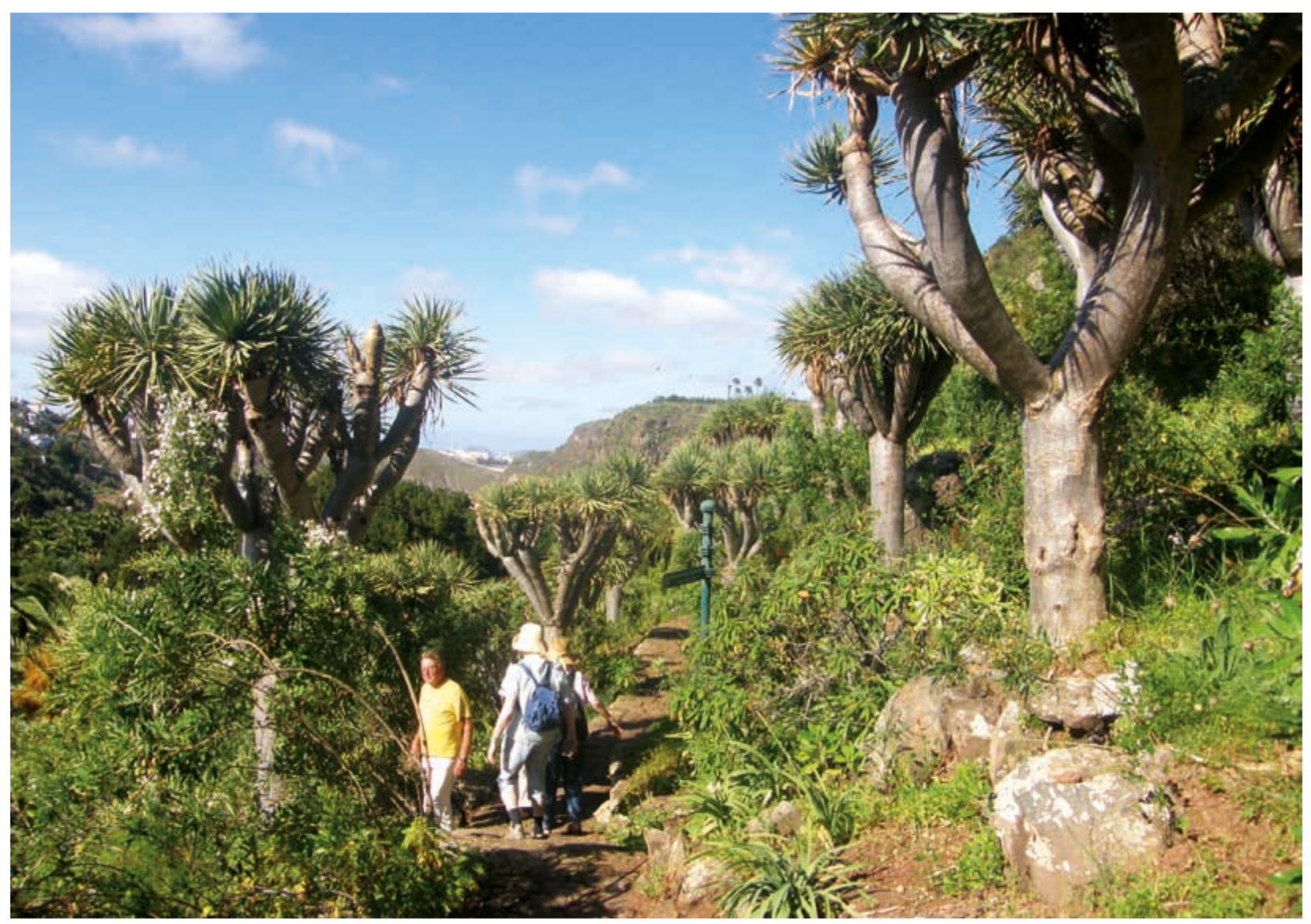

Abb. 7: Drachenbaum-Allee im Botanischen Garten auf Gran Canaria, der von Sventenius angelegt wurde. 
Cruz) ist ein namhafter, mittlerweile emeritierter Botanik-Professor der Universität von La Laguna, der sich in seinem Forscherleben intensiv mit der Flora und Vegetation der Kanaren befasst hat.

Nach Hermann Wildprets Ära wurde der Garten erneut stark vernachlässigt. Im Jahre 1910 merkte Bolleter an: „Hunderte von Forschern und Pflanzenfreunden hat der kleine, liebenswürdige Mann durch den Garten geleitet, voller Freude und Stolz, als derselbe unter seiner Hand herrlich gedieh, voll Wehmut, als er nach seinem Weggang vernachlässigt zu werden begann. Leider mußten auch wir Zeugen sein von dem rasch überhandnehmenden Verfall; herrliche Pflanzen sind wegen Mangel an Pflege zugrunde gegangen; mancherorts wuchert üppiges Unkraut; die Etiketten, mit denen Wild pret seine Pfleglinge versah, sind verschwunden oder verblichen. Man sieht, daß der Garten nicht mehr mit der Liebe gepflegt wird, wie dies von seiten WILDPRETs geschah. Und daß diese Liebe trotz den üblen Erfahrungen bis zu seinem Tode andauerte, beweist ein Brief vom
Sommer 1908, in welchem er seine Freude darüber ausdrückte, daß das Budget des Gartens auf 12000 Pesetas angestiegen sei; er schließt mit den Worten: Wollte Gott, daß der schöne und interessante Garten nun wieder vorwärts kommen werde, was der beste Wunsch seines alten Gärtners ist.“

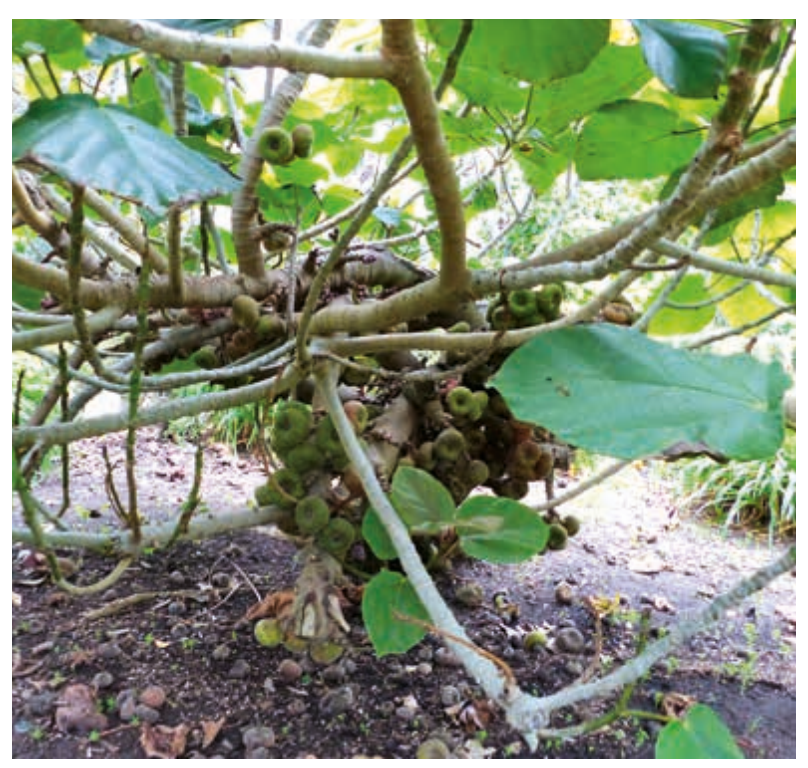

Abb. 8: Ohr-Feige (Ficus auriculata) im Hijuela del Botánico.

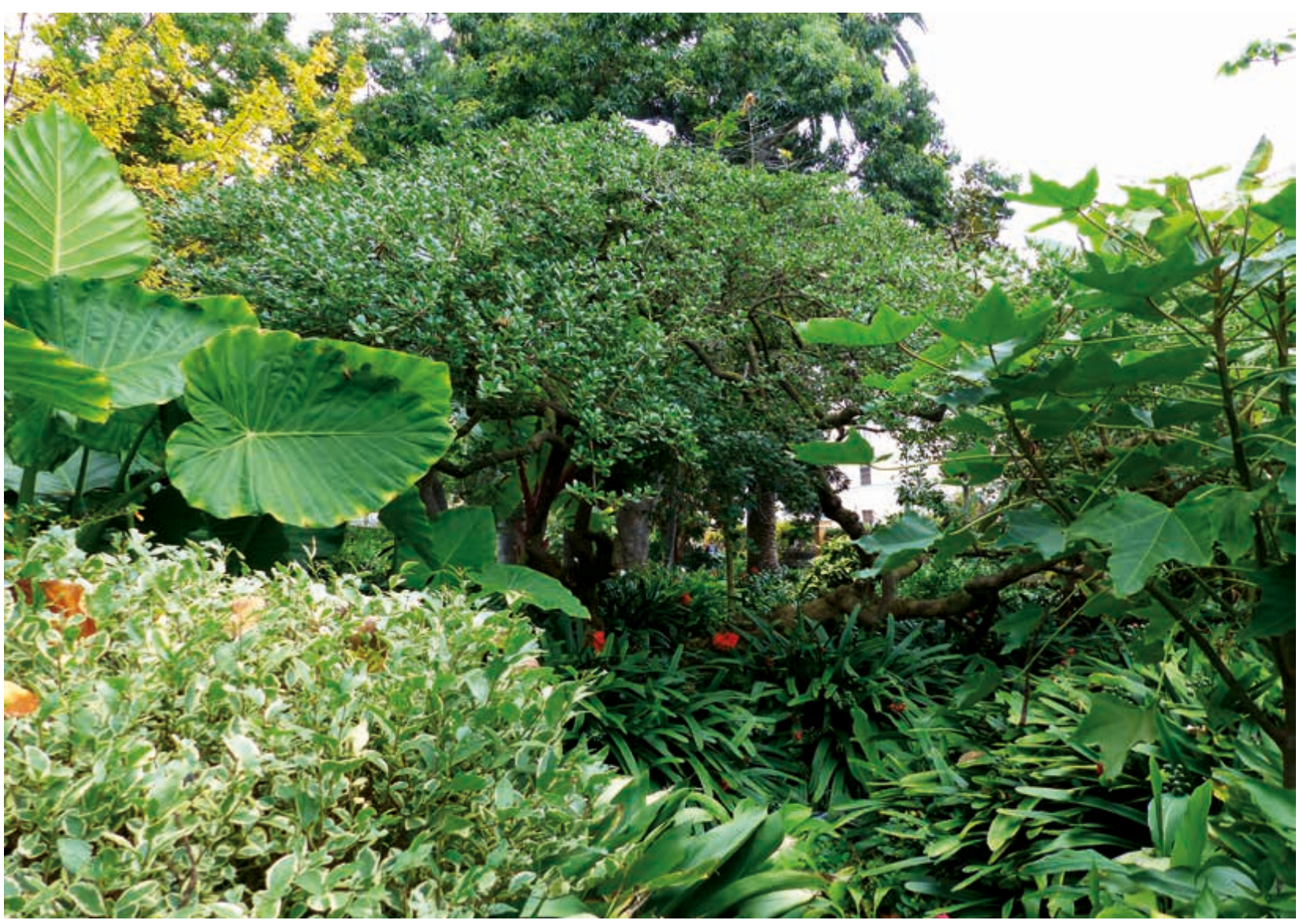

Abb. 9: Blick in den Hijuela del Botánico, einer kleinen Außenstelle des Botánico in Orotava. 


\section{Erik Ragnar Sventenius}

Ein erneuter Aufschwung für den Gartens kam 1943 mit dem schwedischen Botaniker ErIK Ragnar Svensson (latinisiert Sventenius, 1910-1973), der sich hier 20 Jahre lang als Gartenleiter betätigte. Er legte u. a. den Grundstock für ein wissenschaftliches Herbar, das heute rund 40000 Belege umfasst. Sventenius hatte Pläne, den Garten zu erweitern und auszubauen. Da diese nicht umsetzbar waren, kehrte er Teneriffa den Rücken zu und ließ sich auf Gran Canaria nieder, wo er einen neuen Botanischen Garten anlegte. Das Fehlen kontinuierlicher Zuständigkeiten ließ den Garten immer wieder Höhen und Tiefen erleben. Seit 1983 ist er dem Ministerium für Landwirtschaft und Ernährung der Regierung der Kanarischen Inseln unterstellt. Heute ist man sich seiner Bedeutung und dem Wert der alten Pflanzensammlung wieder zunehmend bewusst und man ist darum bemüht, den Garten attraktiv zu erhalten. Außerdem beteiligt sich der Garten an wissenschaftlichen Projekten.

Den wenigsten Besuchern ist vermutlich bekannt, dass der Botánico noch eine kleine Außenstelle hat. Es handelt sich um den Hijuela del Botánico, einen nur $4000 \mathrm{~m}^{2}$ kleinen Garten hinter dem Rathaus in Orotava. Er wurde 1788 auf Veranlassung von Alonso de Nava y Grimón angelegt und enthält neben etlichen buntblühenden Stauden und Bananenpflanzen auch einen alten Kanarischen Drachenbaum, eine sehr mächtige Araucaria heterophylla und eine stattliche Ohr-Feige (Ficus auriculata).

\section{Der Garten und sein Pflanzeninventar}

Neben einer sehr hohen Anzahl an verschiedenen tropischen und subtropischen Nutzpflanzen aus Übersee beherbergt der Botánico auch weitere Schwerpunkte wie z. B. eine Vielzahl an unterschiedlichen Palmen aus ganz verschiedenen Herkunftsregionen. Hierbei ist nicht nur das Artenspektrum der gepflanzten Palmen beeindruckend, sondern auch, dass versucht wurde, Arten aus möglichst vielen unterschiedlichen Gattungen mit ganz unterschiedlichen habituellen Merkmalen zu präsentieren. So sind z. B. Palmengattungen wie Archontophoenix, Attalea, Bactris, Brahea, Butia,

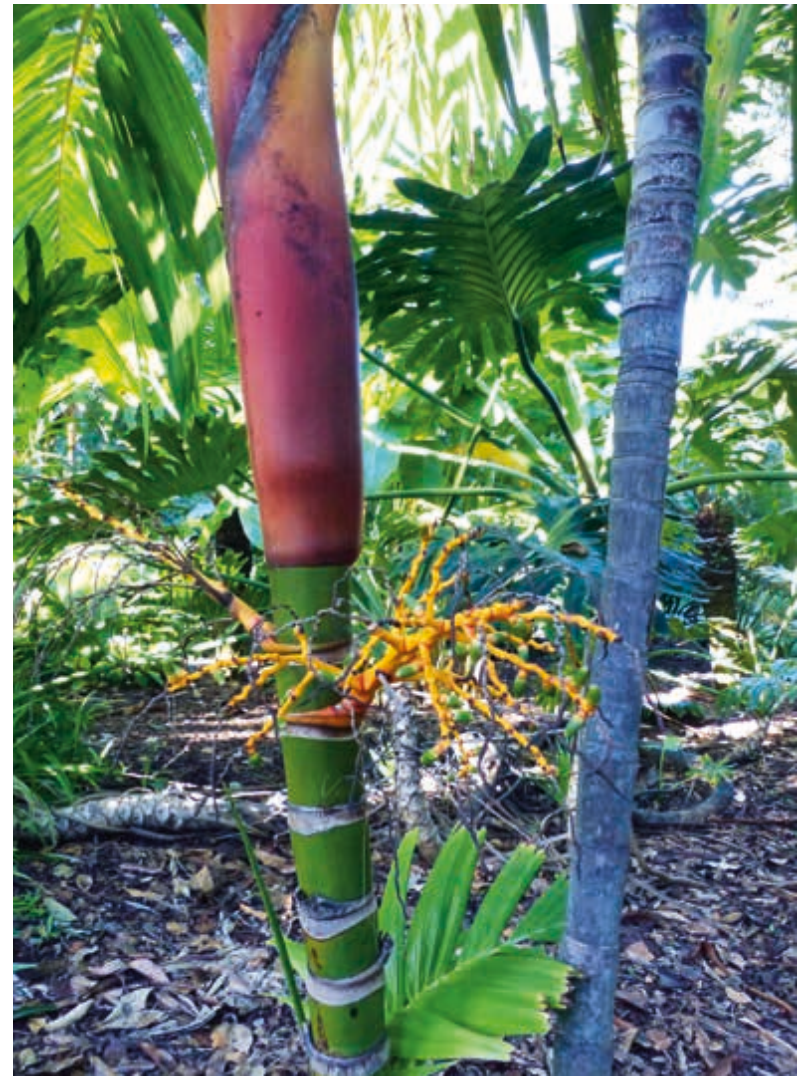

Abb. 10: Areca vestiaria, ein Beispiel für die artenreiche Palmensammlung im Botánico.

Caryota, Chamaedorea, Chamaerops, Dictyosperma, Howea, Hyophorbe, Latania, Pinanga, Ptychosperma, Rhapis, Rhopalostris. Roystonea, Sabal, Salacca, Syagrus, Trachycarpus, Trithrinax, Veitchia oder auch Washingtonia im Garten anzutreffen. Neben den Palmen ist ein weiterer Sammlungsschwerpunkt die Familie der Araceae (Aronstabgewächse). Aus dieser Familie sind zahlreiche verschiedene Gattungen und Arten gepflanzt, die man zwar auch in Deutschland kennt, jedoch bei uns nur als Zimmer- oder Gewächshauspflanzen gedeihen ( $\mathrm{z}$. B. Anthurium oder Zantedeschia). Besonders beeindruckend ist das umfangreiche Artenspektrum an unterschiedlichen Philodendron-Arten, die dort als Unterwuchs gepflanzt wurden und mittlerweile auch regelmäßig blühen (z. B. P. congilaminatum, elegans, giganteum, lacerum, laciniatum, martianum, melanochrysum, longilaminatum, ornatum, panduraeforme, selloum, talamancea, verrucosum, wendlandii, williamsii und $\times$ corsiana). Neben den Bedecktsamern sind auch eine Vielzahl an subtropischen und tropischen Nacktsamern anzutreffen. So steht im Eingangsbereich ein beindruckendes, 
von Tillandsia usneoides überwuchertes Exemplar einer Sumpfzypresse (Taxodium distichum). Unter den baumartigen Nacktsamern ist das riesige Exemplar der Dammaratanne (Agathis dammara, Araucariaceae) der wohl spektakulärste Vertreter. Ansonsten beherbergt der Garten noch eine erwähnenswerte Sammlung an unterschiedlichen, seltenen Palmfarnen wie z. B. Cycas riuminiana, Encephalartos balansae, E. kisambo, E. laurentianus, E. manikensis, Macrozamia miquellii oder auch Lepidozamia peroffskyana. Einige der Palmfarne wie z. B. Cycas circinalis oder Encephalartos altensteinii sind hier mittlerweise zu stattlichen Schopfbäumen herangewachsen. Auch das Thema Farne spielt im Sammlungskonzept eine wichtige Rolle. So sind in und entlang einer schattigen und feuchten Mauer im Eingangsbereich des Gartens zahlreiche Farne aus ebenfalls überwiegend tropischen und subtropischen Herkünfen angepflanzt. Hierzu zählen z. B. Blechnum brasiliensis, Calliopteris prolifera, Goniophlebium subariculatum, Nephrolepis biserrata, Phlebodium aureum, Platycerium alcicorne und Woodwardia radicans.

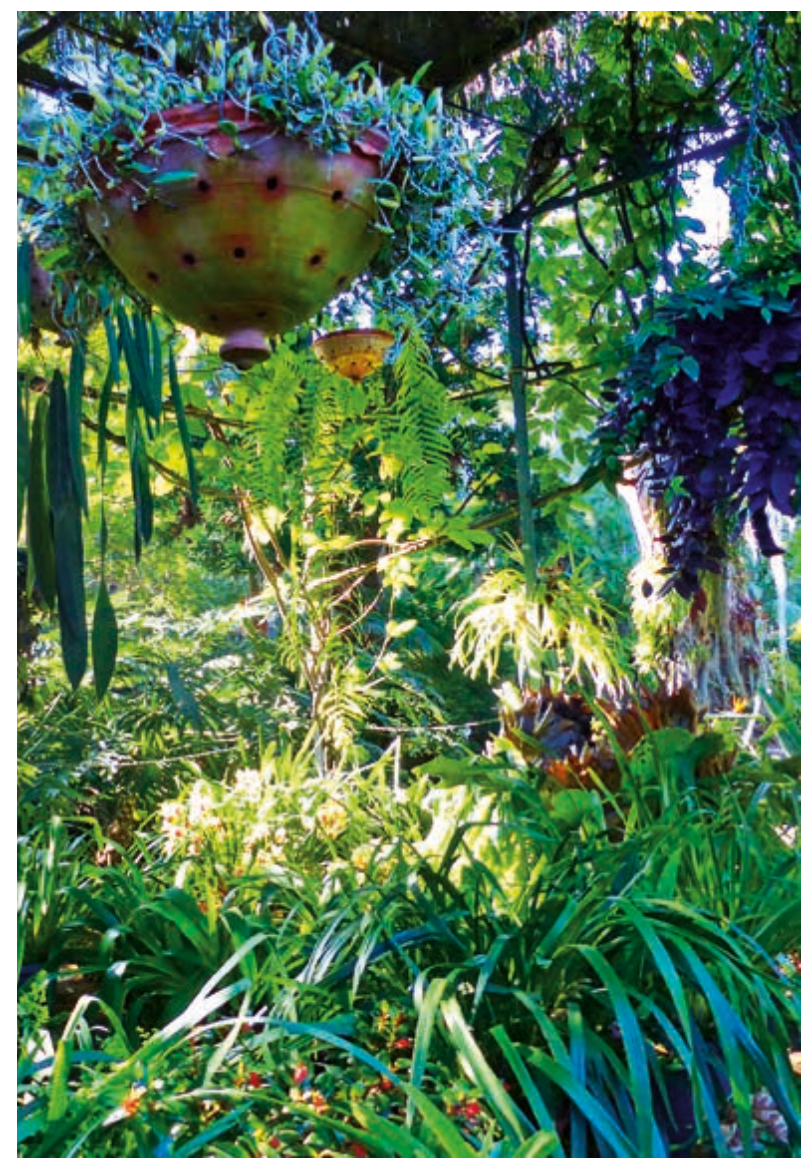

Abb. 11: Schattierter Bereich mit Orchideen und Farnen.

\section{Internetseiten}

Bolleter, E. 1910: Bilder und Studien von einer Reise nach den Kanarischen Inseln. Kapitel 5: HermanN WILDPRET, der Schöpfer des Ruhmes vom botanischen Garten in Orotava. - Leipzig. (online unter http://www.zum.de/ stueber/bolleter/kapitel_05.html.

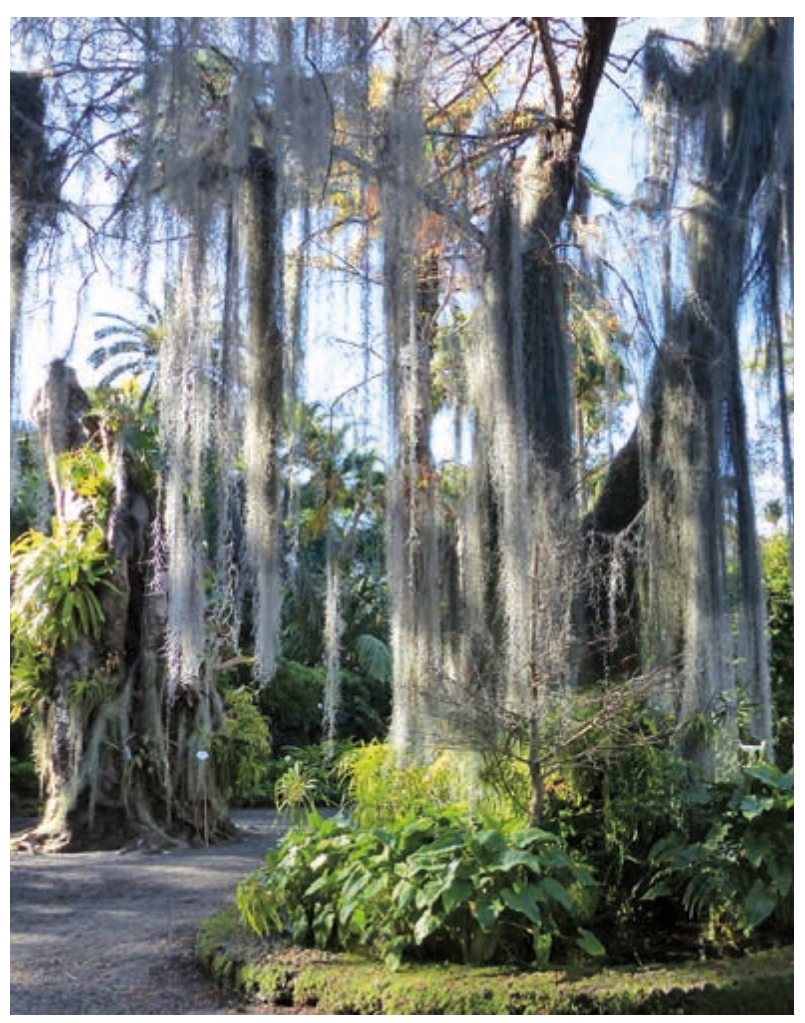

Abb. 12: Sumpfzypresse (Taxodium distichum) mit einem üppigen Behang aus Tillandsia usneoides kurz hinter dem Eingang.

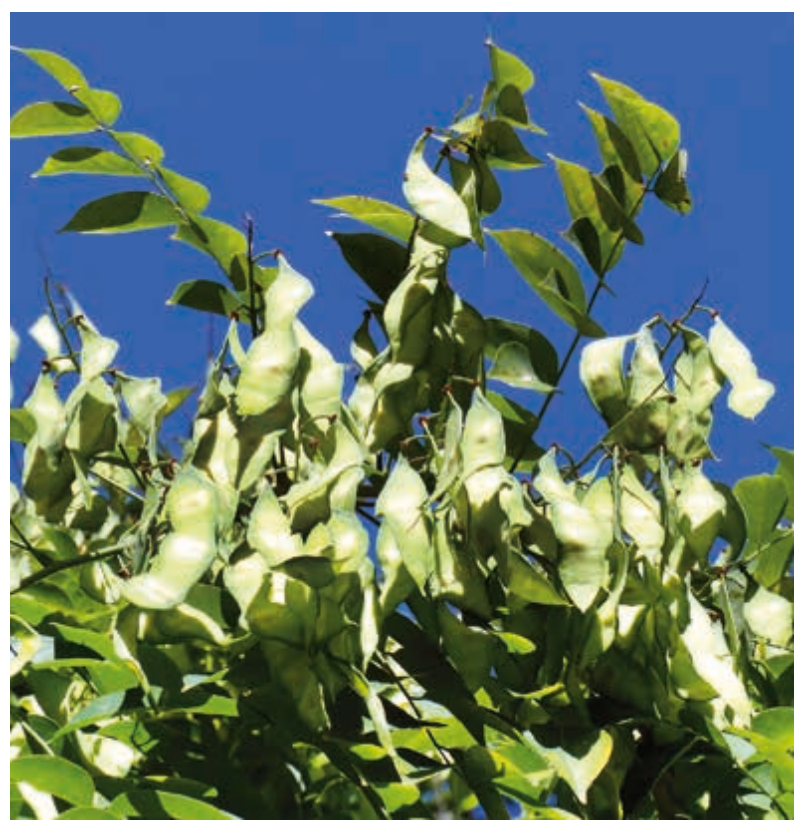

Abb. 13: Fruchtender Zweig des in Mittelamerika heimischen Schmetterlingblütlers Lonchocarpus violaceus. 